\title{
Bistability in a self-assembling system confined by elastic walls: Exact results in a one-dimensional lattice model
}

\author{
J. Pękalski, ${ }^{1}$ A. Ciach, ${ }^{1}$ and N. G. Almarza ${ }^{2}$ \\ ${ }^{1}$ Institute of Physical Chemistry, Polish Academy of Sciences, 01-224 Warszawa, Poland \\ ${ }^{2}$ Instituto de Química Física Rocasolano, CSIC, Serrano 119, E-28006 Madrid, Spain
}

(Received 14 November 2014; accepted 16 December 2014; published online 7 January 2015)

\begin{abstract}
The impact of confinement on self-assembly of particles interacting with short-range attraction and long-range repulsion potential is studied for thermodynamic states corresponding to local ordering of clusters or layers in the bulk. Exact and asymptotic expressions for the local density and for the effective potential between the confining surfaces are obtained for a one-dimensional lattice model introduced by J. Pȩkalski et al. [J. Chem. Phys. 138, 144903 (2013)]. The simple asymptotic formulas are shown to be in good quantitative agreement with exact results for slits containing at least 5 layers. We observe that the incommensurability of the system size and the average distance between the clusters or layers in the bulk leads to structural deformations that are different for different values of the chemical potential $\mu$. The change of the type of defects is reflected in the dependence of density on $\mu$ that has a shape characteristic for phase transitions. Our results may help to avoid misinterpretation of the change of the type of defects as a phase transition in simulations of inhomogeneous systems. Finally, we show that a system confined by soft elastic walls may exhibit bistability such that two system sizes that differ approximately by the average distance between the clusters or layers are almost equally probable. This may happen when the equilibrium separation between the soft boundaries of an empty slit corresponds to the largest stress in the confined self-assembling system. ( 2015 AIP Publishing LLC. [http://dx.doi.org/10.1063/1.4905142]
\end{abstract}

\section{INTRODUCTION}

Competing tendencies in the pair interaction potential often lead to heterogeneity on a mesoscopic length scale. In the case of nanoparticles or globular proteins, there is a competition between solvent-induced attraction and repulsion that is typically (but not exclusively) of electrostatic origin. ${ }^{1-3}$ The effective isotropic short-range attraction (SA) between nanoparticles, ions, or organic molecules favours their aggregation, while the presence of the long-range isotropic repulsion (LR) effects in the separation of the aggregates. The competition between these opposite tendencies results in thermodynamic stabilization of spatially inhomogeneous patterns made of globular or elongated clusters, or layers (stripes in a case of a surface). In ordered phases, the clusters or layers are periodically distributed and form regular patterns. ${ }^{4-7}$ In the disordered phase, the particles also selfassemble into clusters or layers for some range of temperature and concentration. ${ }^{8-10}$ However, in the disordered phase, these objects are ordered only locally, and this short-range order is reflected in the exponentially damped oscillatory behavior of the correlation function on the mesoscopic length scale. ${ }^{4,6,11,12}$

In intracell compartments, in pores of a porous material or on geometrically patterned surfaces, the soft or rigid boundaries of the system can have an ordering or disordering effect on the confined clusters or layers. The key factor is the commensurability of the typical distance between the objects in the bulk and the size of the compartment. Despite the fact that the confinement plays a very important role in biological systems, in pores of porous materials, and on patterned surfaces, the effects of confinement on the self-assembling systems have been much less studied than the bulk properties. In the case of the short-range attraction and long-range repulsion (SALR) interaction potential, the impact of a slittype confinement on thermodynamically stable patterns on a surface ( $2 \mathrm{~d}$ system) was studied by Monte Carlo simulations ${ }^{13}$ and by density functional theory. ${ }^{4}$ In Ref. 13 , the authors found that unlike in the bulk system, in the presence of neutral walls a switch from the cluster to the lamellar morphology with increasing temperature is possible. Moreover, the orientation of the lamella depends on the distance between the walls and the particle-wall interaction parameters. In Ref. 4, the author focused on determining the sequences of stable structures for increasing distance between the walls at a given temperature and for fixed density. He confirmed that the change of the distance can lead to the change of the stable-phase morphology, especially if the period of the structure stable in the bulk and the width of the slit are incommensurate.

In this work, we focus on these effects of confinement on the SALR systems that have not been investigated yet, although in our opinion play a very important role. We limit ourselves to the disordered phase with a short-range order reflected in the oscillatory decay of the correlation function on the mesoscopic length scale. We consider only permeable confining walls, i.e., the system can interchange particles with a reservoir (grand canonical ensemble). Our first question is how the structural defects in the case of incommensurability between the system size and the period in the bulk phase depend on thermodynamic state and on the interaction with the surfaces. The second question concerns 
the fluid-induced effective interactions between the confining surfaces for different values of the chemical potential. The oscillatory decay of the correlation function on the mesoscopic length scale can induce an effective interaction between the confining surfaces that exhibits damped oscillations on the same length scale. This is analogous to the periodic solvation force on the atomic length scale in simple fluids. ${ }^{14,15}$ In contrast to the amphiphilic systems, where the effective interaction was intensively investigated both experimentally ${ }^{16,17}$ and theoretically, ${ }^{18-23}$ in the case of the SALR potential it has not been studied yet.

We consider particles interacting with the SALR potential confined between either rigid or soft walls. The separation between the rigid walls is fixed, while the separation between the soft confining walls can be varied, and this change is associated with elastic energy. The equilibrium separation between the soft walls is determined by the mechanical equilibrium between the solvation force resulting from the stress in the confined self-assembling system, and the elastic force resulting from the deformation of the confining elastic material. We are interested in the question of the equilibrium separation between the elastic walls, especially when the equilibrium thickness of the empty container and the characteristic structural length in the bulk phase are incommensurate.

In order to address the aforementioned questions on a general level, we consider a generic lattice model of a SALR system that can be solved exactly in one dimension (1d). Our results can give some insight for the properties of two and three dimensional systems confined in slits. Moreover, a pseudo-1d system confined by elastic boundaries is formed, for example, by a long protrusion in a vesicle filled with charged nanoparticles. In Ref. 24, it was shown that such a protrusion responds elastically to an external stress in direction parallel to its axis. The bulk properties of the model were thoroughly studied in Ref. 11. In 1d, the ordered phases appear only at $T=0$. The short-range order and the pretransitional effects for $T>0$, however, can be studied based on exact solutions. We found that in the disordered phase, the repulsion between the clusters or layers leads to a dependence of the average density $\rho$ on the chemical potential $\mu$ or pressure $p$ that is significantly different from that of simple fluids. ${ }^{11}$ Characteristic plateaus in $\rho(\mu)$ and $\rho(p)$ appear when the density is equal to the density of the periodic structure that is favoured energetically. This plateau signals that a significant increase of pressure is necessary to overcome the repulsion between the clusters and to compress the system to a dense structure. In addition, for the range of $\mu$ corresponding to the plateau in $\rho(\mu)$, the correlation length is 3 or 4 orders of magnitude larger than the size of the particles. ${ }^{11}$ When the range of correlations is so large, the disordered and the ordered phases can behave in a very similar way in slits of a width much smaller than the correlation length, and larger than the size of the cluster. The shape of the $\rho(p)$ curve and the large correlation length suggest that the solvation force can be quite strong even for large separations between the confining surfaces. We shall verify this expectation by exact results.

The bulk structure can be deformed not only by the physical confinement but also in a simulation box when its size is incommensurate with the period of the phase with long- or short-range order. This incommensurability is a serious issue when the period of the bulk phase is unknown. To overcome this problem, successful but computationally demanding method has been developed in Ref. 25. Later an elegant and easier method was proposed in Ref. 26. Instead of introducing another simulation method, in this work, we show how to avoid misinterpretation of the results of the standard Monte Carlo simulations for $\rho(\mu)$. Our exact results directly show the effect of the incommensurability on the shape of $\rho(\mu)$.

In Sec. II, we briefly describe the model introduced in Ref. 11 and the transfer matrix method. The details of the derivations are described in the Appendices. In Sec. III, we present asymptotic expressions for the local density and for the effective potential between the confining surfaces. We determine the range of validity of these formulas by comparison with exact results. In Sec. IV, we discuss the dependence of the distribution of the particles inside the pore on the chemical potential when the width of the slit and the period of the bulk structure are incommensurate. We also compare the shape of $\rho(\mu)$ for various slits with the result obtained in Ref. 11 for the bulk. In addition, we consider periodic boundary conditions (PBC) in the case of incommensurability, in order to help to interpret simulation results. The effective interaction between the walls for different thermodynamic states and the equilibrium width of a system with elastic boundaries are determined in Sec. V. The summary and conclusions are presented in Sec. VI.

\section{THE MODEL AND THE METHOD OF EXACT SOLUTIONS}

We consider a lattice model for systems with particles interacting with a SALR potential. We assume that the particles occupy lattice sites on a 1 dimensional lattice, and the lattice constant $a$ is comparable with the particle diameter $\sigma$. The particles attract or repel each other when they are the nearest or the third neighbors, respectively. Such interactions lead to formation of small clusters and characterize, for example, cone-shape membrane proteins or globular proteins in solvents with weak ionic strength. In particular, similar ranges of interactions were found for lysozyme molecules in water. ${ }^{3} \mathrm{We}$ discuss the model in more detail in Ref. 12. The model with the same interaction potential and with PBC was solved exactly in Ref. 11 for the system sizes $L=6 N$, where $N$ is integer. For such system sizes, the energetically favourable structure of 3 occupied sites separated by 3 empty sites is possible, and the properties of the bulk system can be reproduced. For $L \neq 6 N$, the incommensurability between the system size $L$ and the period of the ordered structure may influence the results. Here, we focus on the impact of this incommensurability in the case of the PBC and in the case of a slit type of confinement, i.e., with rigid boundary conditions (RBC). In the case of the PBC, we ask how the incommensurability influences the dependence of the density $\rho$ on the chemical potential $\mu$ for different system sizes. Our exact results may help to interpret the results of simulations that are performed for finite systems, and the incommensurability is a serious problem. In the case of 


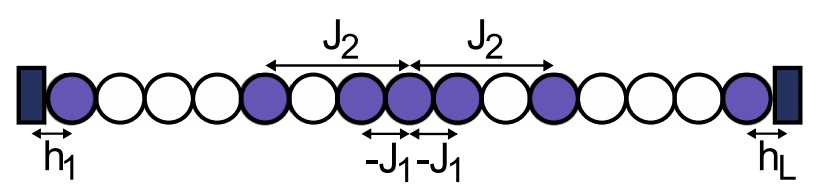

FIG. 1. Scheme of the model for a system of size $L=15$. The lattice constant $a$ is equal to the particle diameter $\sigma$. The particles attract or repel each other with the energy $-J_{1}$ or $J_{2}$ when they are the nearest or the third neighbors, respectively. If a particle occupies the first or the last site of the lattice, then it interacts with the confining wall with the energy $h_{1}$ or $h_{L}$, respectively.

the RBC, we assume that the confining walls are electrically neutral, hence they interact only with the particles located at the first and the last site of the system (see Fig. 1). The confining walls represent real physical confinement, e.g., in a porous material or in a thin film on a solid substrate.

We assume that the lattice consists of $L$ sites labeled from 1 to $L$. In order to describe whether the lattice site $x$ is occupied or not, we introduce an occupation operator $\hat{\rho}(x)$ which is equal to 1 or 0 , respectively. Hence, the configuration of the system (the microstate) is given by $\{\hat{\rho}(x)\} \equiv(\hat{\rho}(1), \ldots, \hat{\rho}(L))$. The probability of the microstate $\{\hat{\rho}(x)\}$ is

$$
\mathbb{P}[\{\hat{\rho}(x)\}]=\frac{e^{-\beta H[\{\hat{\rho}(x)\}]}}{\Xi},
$$

where $\Xi$ is the Grand Partition function, $\beta=\left(k_{B} T\right)^{-1}, k_{B}$ is the Boltzmann constant, and $H$ is the thermodynamic Hamiltonian which contains the energy and the chemical potential term

$$
\begin{aligned}
H[\{\hat{\rho}\}]= & \frac{1}{2} \sum_{x=1}^{L} \sum_{x^{\prime}=1}^{L} \hat{\rho}(x) V\left(x-x^{\prime}\right) \hat{\rho}\left(x^{\prime}\right) \\
& +h_{1} \hat{\rho}(1)+h_{L} \hat{\rho}(L)-\mu \sum_{x=1}^{L} \hat{\rho}(x),
\end{aligned}
$$

where the particle-particle interaction potential is

$$
V(\Delta x)= \begin{cases}-J_{1} & \text { for }|\Delta x|=1, \\ +J_{2} & \text { for }|\Delta x|=3, \\ 0 & \text { otherwise }\end{cases}
$$

We choose $J_{1}$ as the energy unit and introduce dimensionless variables for any quantity $X$ with dimension of energy as $X^{*}=X / J_{1}$, in particular,

$$
\begin{aligned}
& T^{*}=k_{B} T / J_{1}, \quad J^{*}=J_{2} / J_{1}, \quad h_{1}^{*}=h_{1} / J_{1}, \\
& h_{L}^{*}=h_{L} / J_{1}, \quad \mu^{*}=\mu / J_{1} .
\end{aligned}
$$

We solve the model exactly by the transfer matrix method, as in Ref. 11. Similar transfer matrix method was used before in the case of the $1 \mathrm{~d}$ axial next-nearest-neighbor Ising (ANNNI) model, with ferromagnetic interaction between the nearest neighbour spins, and antiferromagnetic interaction between the spins that are next neighbours. ${ }^{27}$ There is a unique mapping between our model and the model with competing ferromagnetic and antiferromagnetic interactions between the first and the third neighbours in a presence of magnetic field. In our case, the range of interaction is larger than in the 1d ANNNI model, and this leads to a larger dimension of the transfer matrix. Because the range of the particle-particle interaction is 3 , the transfer matrix operates between the microstates in boxes that are located next to each other, and each box consists of 3 sites. There are 8 microstates in each box, therefore the dimension of the transfer matrix is 8 . The system can be divided into such boxes when $L=3 N$. In general, the expression for the grand partition function for $L=3 N+j$ depends on both, $N$ and the reminder of division of $\mathrm{L}$ by 3 , $j=L \bmod 3=0,1,2$. We describe the method in more detail, and give the exact expression for $\Xi$ in Appendix A.

When the correlation length between the particles is comparable with the distance between the confining walls, then the distribution of the particles is influenced by both walls. This leads to the excess of the grand potential depending on the distance between the walls, ${ }^{28}$

$$
\Omega_{e x} \equiv \Omega-\Omega_{b u l k}=\gamma_{1}+\gamma_{L}+\Psi(L),
$$

where $\Omega=-k_{B} T \ln \Xi$ and $\Omega_{\text {bulk }}=-k_{B} T \ln \Xi_{\text {bulk }}$ are the grand potential in the slit and in the bulk of the same size $L$, respectively, $\gamma_{1}$ and $\gamma_{L}$ are the wall-fluid surface tensions, and $\Psi(L)$ corresponds to the effective interaction between the confining walls. ${ }^{28}$ The effective force between the surfaces is $-\nabla \Psi(L)$. The exact expressions for $\gamma_{1}, \gamma_{L}$, and $\Psi(L)$ are given in Appendix C.

The expression for the local average density at the site $x=3 n+l$ in the system of size $L=3 N+j$ with $j=0,1,2$ depends on both, $n$ and $l=1,2,3$, and in addition on $N$ and $j$. The rather complex formulas are given in Appendix B. The exact expressions take much simpler asymptotic forms for $N \gg 1$ and $n \simeq N / 2$. We present the asymptotic formulas for $N \gg 1$ for the density and for $\Psi(L)$, and compare them with the exact results in Sec. III.

\section{ASYMPTOTIC EXPRESSIONS FOR LARGE SLITS AND THE RANGE OF THEIR VALIDITY}

In the energetically favourable structure, clusters composed of 3 particles are separated by 3 empty sites. For this reason, the properties of the system confined in the slit of large width $L=3 N+j$ depend on both, the number of the triples of sites, $N \approx L / 3$, and the number of the additional sites, $j=0,1,2$. Let us first consider the average local density in the central part of the slit of large width, $N \gg 1$. We divide the system into triples of sites. Each site $x$ is characterized by the number of the triple to which it belongs, $n$, and the position inside the triple, $l$, so that $x=3 n+l$ with $l=1,2,3$. The expression for $\langle\hat{\rho}(3 n+l)\rangle$ depends on $n$ and $l$, as well as on $N$ and $j$. From the exact formulas given in Appendix B, we obtain the asymptotic expression for $N \rightarrow \infty$ and $n \simeq N / 2$ (central part of the slit),

$$
\begin{aligned}
\langle\hat{\rho}(3 n+l)\rangle \simeq & \bar{\rho}+A_{1}(l) \cos \left(n \lambda+\theta_{1}(l)\right) e^{-3 n / \xi} \\
& +A_{L}(l) \cos \left((N-n) \lambda+\theta_{L}(l)\right) e^{-3(N-n) / \xi}
\end{aligned}
$$

The explicit expressions for $\bar{\rho}$, the amplitudes $A_{1}(l), A_{L}(l)$, and the phases $\theta_{1}(l), \theta_{L}(l)$ are given in Appendix B (these quantities depend also on $N$ and $j$ ). The decay length $\xi$ is given by the same expression as the correlation length in the bulk, ${ }^{11}$

$$
\xi=3 / \ln \left(\frac{\lambda_{1}}{\left|\lambda_{2}\right|}\right)
$$


where $\lambda_{1}$ and $\lambda_{2}=\left|\lambda_{2}\right| \exp (i \lambda)$ are the largest and the second largest eigenvalues of the transfer matrix. The transfer matrix is not Hermitian, and some of the eigenvalues can be complex. The presence of the imaginary part of $\lambda_{2}$ depends on $J^{*}$, and on the thermodynamic state. The monotonic decay of the density near a single surface occurs when $\lambda_{2}$ is real and positive $(\lambda=0)$. The exponentially damped periodic structure with the period 6 occurs when $\lambda_{2}$ is real and negative $(\lambda=\pi)$. In most cases, however, including $J^{*}=3$ for the range of $\mu^{*}$ studied in this work, $\lambda_{2}$ is complex and the period of the damped oscillations is noninteger.

The asymptotic formula for the effective interaction potential for $N \rightarrow \infty$ is

$$
\beta \Psi(3 N+j) \simeq A(j) \cos (\lambda N+\phi(j)) e^{-3 N / \xi} .
$$

The explicit expressions for the amplitude $A(j)$ and the phase $\phi(j)$ are given in Appendix C.

The asymptotic formulas are simply the exponentially damped periodic functions. Similar expressions, but without the amplitude modulations, were obtained in mean-field theories of confined self-assembling systems..$^{20,29-31}$ These rather simple asymptotic forms are strictly valid for $N \gg 1$ and $n \simeq N / 2$. We check the validity of the asymptotic expressions by comparing them with the exact results. The exact and asymptotic formulas are valid for any $J^{*}$. To fix attention, we focus in this paper on the case of strong repulsion, $J^{*}=3$.

As shown in Fig. 2, the agreement of the asymptotic expression for the local density with the exact result is very good already for $L=42$, and the discrepancy between the exact and asymptotic expressions appears only very close to the surface. For $L=30$, the accuracy of the asymptotic expression is less good but it is still satisfactory, except from the clusters adsorbed at the surfaces, where some discrepancy can be observed. Thus, the asymptotic formula is sufficiently accurate not only in the center, but inside the whole slit for slits containing 5 or more clusters.

In the asymptotic expressions, the decay length and the period of oscillations of the local density in the slit, and the correlation function in the bulk are the same. In Fig. 3, we
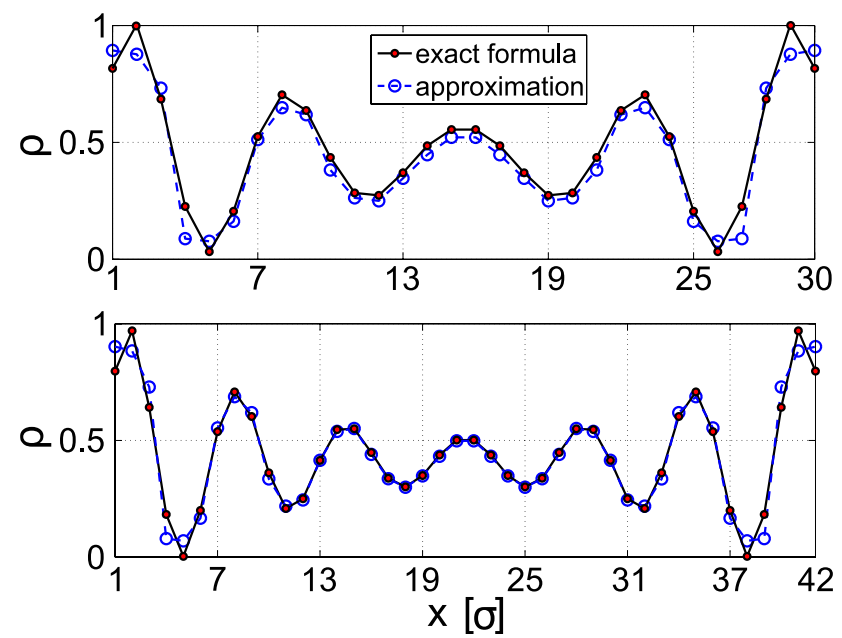

FIG. 2. Comparison of the exact (B2) and approximate (7) formulas for the average density for $J^{*}=3, \mu^{*}=0, T^{*}=0.5$, and $h_{1}^{*}=h_{L}^{*}=-1$. Upper panel $L=30$, lower panel $L=42$.

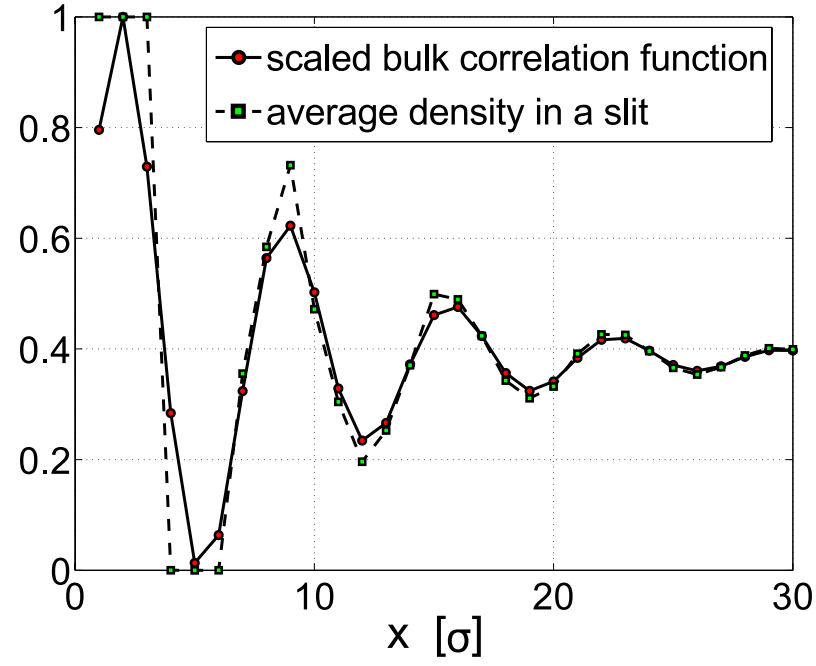

FIG. 3. Comparison of the density profile in a slit (dashed line) for $J^{*}=3$, $\mu^{*}=-0.66, T^{*}=0.0125, h_{1}^{*}=h_{L}^{*}=-1$, and $L=96$, and the bulk correlation function obtained in Ref. 11 (solid line) for the same thermodynamic state. The correlation function was linearly scaled and shifted by the average density of the bulk system, $\rho=0.3841$.

compare the exact results for the local density and for the correlation function. In order to compare the two functions, we add the average density of the bulk system to the linearly scaled correlation function, and obtain good agreement for the distance from the surface $x>10$. We conclude that the correlation function in the bulk describes very well the local structure (up to an amplitude that depends on the kind of the wall) except for the first cluster adsorbed at the surface.

In Fig. 4, the exact and approximate results for the effective interaction between the walls are compared. As expected, the accuracy of the asymptotic formula improves with increasing system size. Close to the minima, i.e., near the equilibrium separations between the surfaces, the approximate formula works well also for small systems. On the other hand, for small and incommensurate system sizes, the approximate formula highly underestimates the interaction potential, therefore for small systems it underestimates the effective force between the walls.

Notice that for some system sizes there is no clear minimum and $\Psi(L)$ takes almost equal values for two

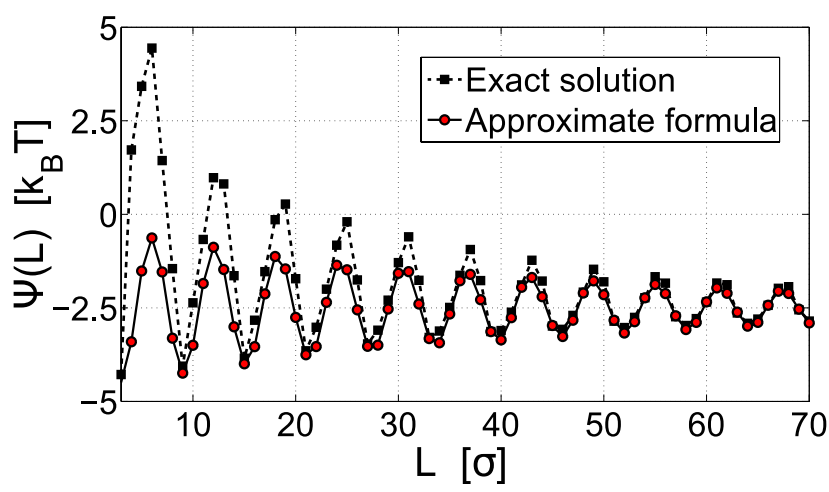

FIG. 4. The effective wall-wall interaction $\Psi(L)$ for $J^{*}=3, \mu^{*}=0$, $T^{*}=0.2$, and $h_{1}^{*}=h_{L}^{*}=-1$. Dashed line with squares - the exact formula, Eq. (C6). Solid line with circles - the approximate formula, Eq. (9). 
consecutive system sizes. However, for the exact and the approximate formulas, this phenomenon occurs for different system sizes, e.g., in Fig. 4 for $L=39$, 40 for the exact result, and for $L=27,28$ for the approximate formula.

\section{EFFECTS OF INCOMMENSURABILITY OF THE SYSTEM SIZE AND THE PERIOD OF THE BULK STRUCTURE}

In this section, we study the effect of the incommensurability of the system size and the period of the bulk structure on the distribution of the particles and on the dependence of the average density on the chemical potential. Our aim is to verify how $\rho\left(\mu^{*}\right)$ is influenced by the presence of structural defects that must be present in the case of the incommensurability. We first consider the PBC, and next the RBC.

\section{A. The case of PBC}

We focus on $L=6 N+3$, i.e., on the largest mismatch between $L$ and 6 (the low- $T$ period in the ordered phase). Let us first investigate the GS, i.e., the case of $T^{*}=0$. For $L=6 N+3$, we may expect that in the periodic phase either a separation between some clusters is larger than 3 , or some clusters are larger than 3 (see Fig. 5). When the separation between the clusters is larger than 3 and we add one particle to a cluster consisting of at least 3 particles, then the increase of the Hamiltonian is $\Delta H^{*}=-1+J^{*}-\mu^{*}$. For $\mu^{*}<J^{*}-1$ or $\mu^{*}>J^{*}-1$, we have $\Delta H^{*}>0$ or $\Delta H^{*}<0$, respectively. Thus, in the GS corresponding to the minimum of the Hamiltonian $H^{*}$, the voids in the first case and the clusters in the second case occupy 3 more sites. At $T^{*}=0$, the average density jumps by $3 / L$ for $\mu^{*}=J^{*}-1$. The GS in the bulk $(L=6 N)$ and for $L=9$ is shown in Fig. 5 for $J^{*}=3$. Note that we have $\Delta H^{*}=-1+J^{*}-\mu^{*}=0$ for $\mu^{*}=2$ in this case, therefore for $\mu^{*}=2$ the GS is degenerate, and the cluster can consist of either 3, 4, 5, or 6 particles.

In Fig. 6, we show $\rho\left(\mu^{*}\right)$ for $T^{*}=0.15$. Note that the transition between the two types of defects, i.e., larger voids or larger clusters could be misinterpreted as a transition between different phases, because when a system undergoes a firstorder phase transition, steps in $\rho\left(\mu^{*}\right)$ appear. The results of

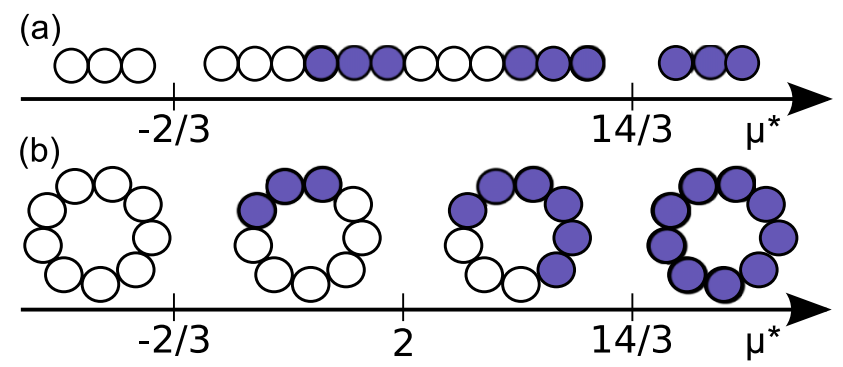

FIG. 5. Schematic representation of the ground state $(\mathrm{GS})\left(T^{*}=0\right)$ for $J^{*}=3$ for the bulk system $(L=6 N)$ (a) and for the system of size $L=9$ (b). For $\mu^{*}<-2 / 3$ or $\mu^{*}>14 / 3$, the stable phase is the vacuum or the fully occupied lattice. For $-2 / 3<\mu^{*}<14 / 3$, the periodic phase is stable in the bulk. For $L=9$, the stability region of the periodic phase is split into $\mu^{*}<2$ and $\mu^{*}>2$ corresponding to enlarged void or cluster, respectively.

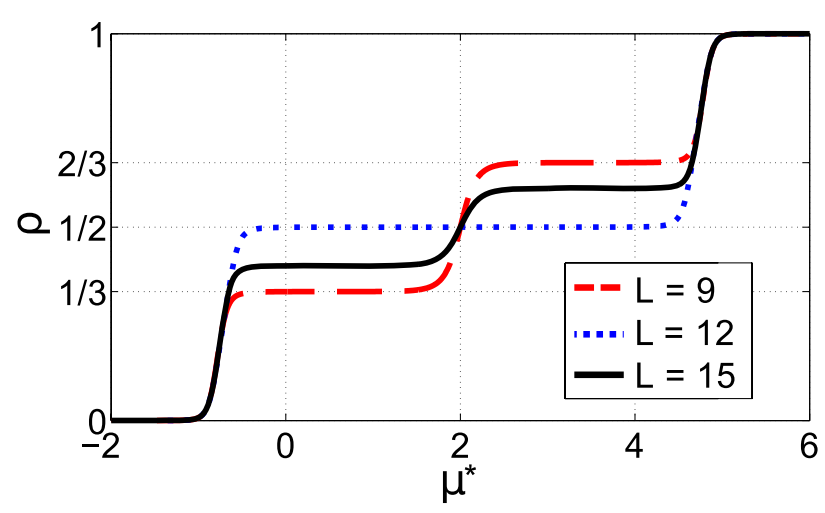

FIG. 6. The average density $\rho^{*}$ as a function of the chemical potential $\mu^{*}$ for $J^{*}=3$ and $T^{*}=0.15$, for a system with PBC and $L=9$ (red dashed line), $L=12$ (blue dotted line), and $L=15$ (black solid line).

simulations in the case of systems with spatial inhomogeneities on a mesoscopic length scale should be interpreted with special care, especially when several periodic phases with different periods can appear. Our results show that in the case of structural defects, the height of the step decreases as $\sim 1 / L$ for increasing $L$, and for certain system sizes the step disappears (Fig. 6).

\section{B. The case of RBC}

We first focus on attractive surfaces. Let us consider the Hamiltonian for a single cluster composed of $n \leq 3$ particles adsorbed at the surface, $H^{*}=h_{1}^{*}-(n-1)-n \mu^{*}$. The adsorption of the cluster is energetically favourable compared to vacuum for $\mu^{*}>\left(h_{1}^{*}+1-n\right) / n$. In order to fix attention, we assume that the interaction with the surfaces is the same as the particle-particle attraction, $h_{1}^{*}=h_{L}^{*}=-1$. In this case, a cluster adsorbed at each attractive surface is energetically favourable for $\mu^{*}>-1$. Thus, for $\mu^{*}>-1$, the largest mismatch between the system size and the structure of the bulk periodic phase occurs for $L=6 N$ when both surfaces are attractive. For $L \neq 6 N+3$, the GS of the system is degenerate in the whole stability region of the periodic phase, because the defects in the periodic structure that are caused by the incommensurability of the period and the system size are not localized. Moreover, the stability region of the periodic phase splits into 4 regions, corresponding to different numbers and sizes of the clusters present in the slit. We choose $L=19$ and present typical microscopic states of the GS in Fig. 7. For $\mu^{*}<0$, there are 3 clusters in the slit. Each of them consists of 3 particles, and the neighboring clusters are separated by at least 3 empty sites. Apart from this limitation the position of the central cluster can be arbitrary. For $0<\mu^{*}<J^{*}-1$, there are 2 clusters consisting of 3 particles and 2 clusters consisting of 2 particles in the slit. The clusters do not repel each other, i.e., there are 3 empty sites between the neighboring clusters. For $J^{*}-1<\mu^{*}<2\left(J^{*}-1\right)$, there are 4 clusters consisting of 3 particles. Finally, for $\mu^{*}>2\left(J^{*}-1\right)$, there are 3 clusters separated by two voids composed of 3 empty sites, and each cluster consists of at least 3 particles.

In Fig. 8, we present $\rho\left(\mu^{*}\right)$ for the slit with $L=19$ at $T^{*}=0.15$. The average densities corresponding to the plateaus are shown in the insets. Note the similarity between the average 
(a)

(b)

(c)

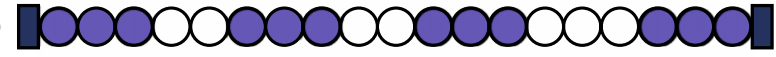

(d)

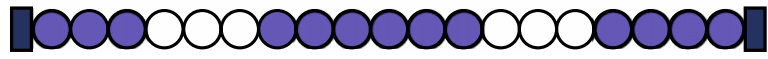

FIG. 7. Typical microstates in the degenerate GS for a slit of size $L=19$ with attractive walls. The range of the chemical potential corresponding to the shown microstates is (a) $-2 / 3<\mu^{*}<0$, (b) $0<\mu^{*}<J^{*}-1$, (c) $J^{*}-1<\mu^{*}<2\left(J^{*}-1\right)$, and (d) $2\left(J^{*}-1\right)<\mu^{*}<2 J^{*}-4 / 3$.

densities in the GS (a)-(d) (Fig. 7) and the insets (a)-(d) in Fig. 8.

In the case of confinement in a slit, the steps in $\rho\left(\mu^{*}\right)$ represent a physical effect, namely, structural changes such as a jump of a number of the clusters or a change of their size as a function of the chemical potential. Such abrupt changes in a slit induced by small changes in the surroundings occur when the size of the system and the period of the bulk phase are incommensurate.

Let us focus on the role of the interaction with the confining surfaces. The attractive and repulsive surfaces are compared in Fig. 9 for a large slit. When the walls are attractive, we observe steps in $\rho\left(\mu^{*}\right)$ associated with adsorption of a particle or a cluster. The first step is present only if $h_{1}^{*}, h_{L}^{*}<-1$ and it originates from the adsorption of a single particle at each wall, while the second step corresponds to the adsorption of clusters. Note that for $\mu^{*}>-1$ the $\rho\left(\mu^{*}\right)$ curves are essentially the same for $h_{1}^{*}=h_{L}^{*}=-1$ and $h_{1}^{*}=h_{L}^{*}=-1.5$. This shows that for strong wall-fluid attraction, the results are independent of $h_{1}^{*}$ and $h_{L}^{*}$.

In the case of short-range interactions with the walls, the density profiles in the slits with attractive and repulsive surfaces are very similar for $-0.75<\mu^{*}<-0.5$ (Fig. 9(b)). This rather surprising property follows from the fact that even though the clusters do not touch the repulsive surfaces, they are located very close to them. A significant difference between the

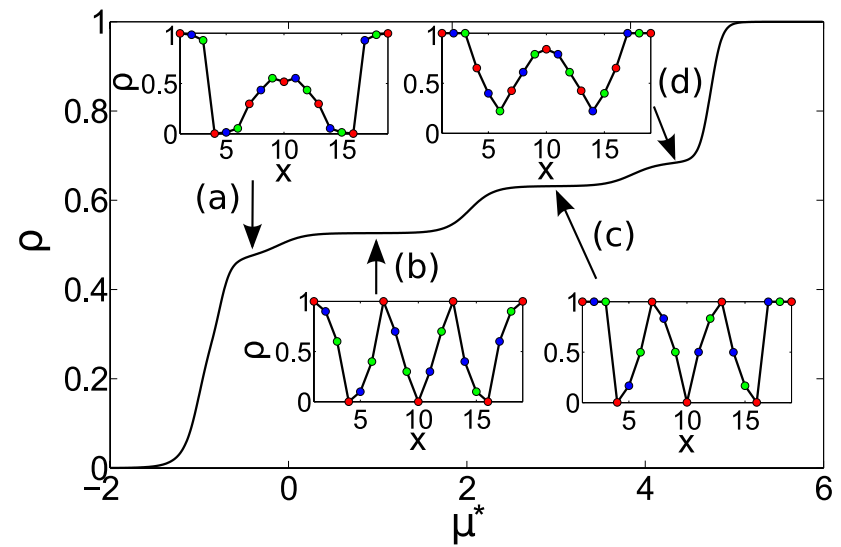

FIG. 8. Density $\rho$ as a function of the dimensionless chemical potential $\mu^{*}$ for $J^{*}=3$ and $T^{*}=0.15$ in a slit of size $L=19$ with attractive walls. For increasing $\mu^{*}$, we observe 4 plateaus. The plateaus from (a) to (d) correspond to the average densities shown in the insets. The steps between them occur for $\mu^{*} \approx 0,2,4$, i.e., near the GS coexistence between different structures in confinement (see Fig. 7). (a)

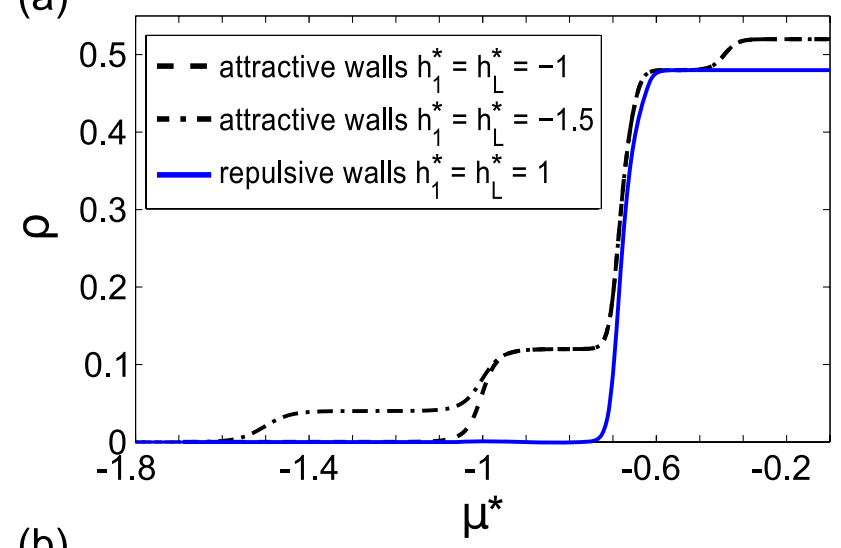

(b)
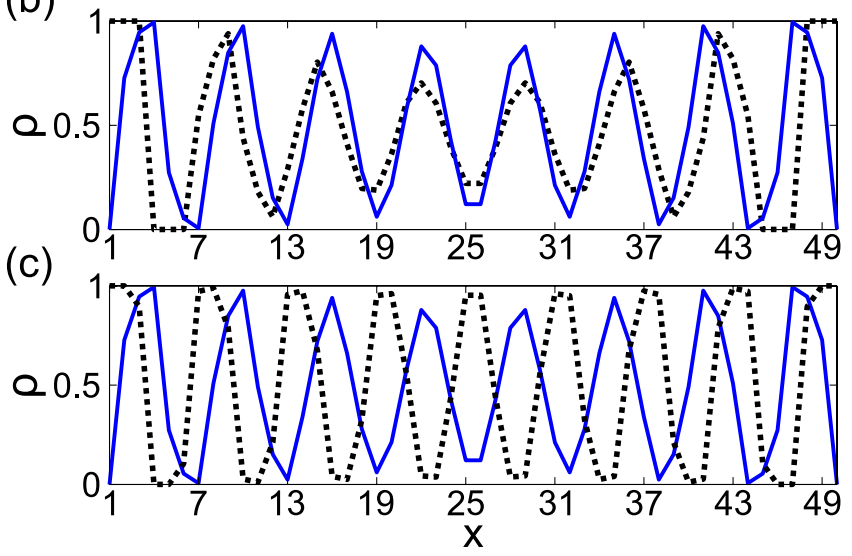

FIG. 9. Panel (a) $\rho\left(\mu^{*}\right)$ for $J^{*}=3, T^{*}=0.03$, and $L=50$ for systems with $\mathrm{PBC}, \mathrm{RBC}$ with attractive walls for $h_{1}^{*}=h_{L}^{*}=-1$ (dashed line) and for $h_{1}^{*}=h_{L}^{*}=-1.5$ (dashed-dotted line), and RBC with repulsive walls for $h_{1}^{*}=h_{L}^{*}=1$ (solid line). The rapid changes of the density at $\mu^{*} \approx-1.5$ and at $\mu^{*} \approx-1$ correspond to the adsorption of a particle and a cluster, respectively, at the attractive walls. Panels (b) and (c) show density profiles for $\mu^{*}=-0.55$ and $\mu^{*}=-0.4$, respectively, for attractive (dotted lines) and repulsive (solid lines) surfaces. Note the change of the number of clusters for $\mu^{*} \approx-0.45$ when the walls are attractive.

attractive and repulsive surfaces appears only for $\mu^{*}>-0.45-$ there is one more cluster, and one more step in $\rho\left(\mu^{*}\right)$ in the slit with the attractive surfaces for $L=20,26,32, \ldots$.

\section{EFFECTIVE INTERACTION BETWEEN THE WALLS AND DEFORMATIONS OF ELASTIC CONTAINERS}

In this section, we discuss the effective potential between the confining surfaces separated by the distance $L$. We first consider walls separated by a fixed distance. Next we assume that the walls are elastic, and the change of the wall separation is possible at the cost of elastic energy. When the equilibrium width of the empty slit, $L_{0}$, and the period of the ordered phase do not match, the elastic energy and the fluid-induced stress are in competition. We ask how the equilibrium width of the slit filled with the inhomogeneous fluid differs from $L_{0}$.

\section{A. The case of fixed distance between the confining walls}

The exact results for the effective potential between the confining walls separated by a fixed distance, $\Psi(L)$, are presented in Fig. 10 for the chemical potential corresponding 

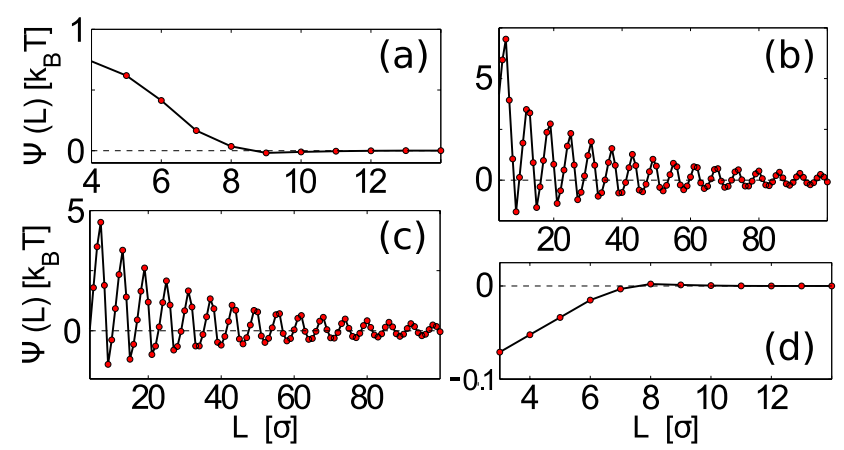

FIG. 10. $\Psi(L)$ for $J^{*}=3$ and $T^{*}=0.2$ for different values of the chemical potential $\mu^{*}$ and both walls attractive. (a) $\mu^{*}=-1$, (b) $\mu^{*}=0$, (c) $\mu^{*}=4$, and (d) $\mu^{*}=5$. $L$ is in units of the particle diameter $\sigma$.

to the GS stability of the vacuum, the periodic phase, and the dense phase (compare Fig. 5). Note that the confined fluid leads to repulsion or attraction between the walls when the dilute or the dense pseudo-phase is stable in the bulk, respectively. The repulsion may follow from the adsorption of the clusters at the surfaces, since the clusters repel each other. The oscillations of $\Psi(L)$ are present if the periodic distribution of clusters is thermodynamically preferred. These oscillations should be interpreted as follows: the minima of $\Psi(L)$ correspond to the system sizes commensurate with the periodic structure, therefore if we would allow the system to shrink or expand, then in order to suppress the internal stress the system would change its size to the value corresponding to the nearest minimum of $\Psi(L)$. The bigger is the slope of the oscillations, the stronger is the effective force leading to the nearest minimum of $\Psi(L)$.

For large $L$, the decay rate of $\Psi(L), \xi$, is equal to the bulk correlation length (see Eq. (9)). In Ref. 11, it was shown that the correlation length in the considered model can be a few orders of magnitude larger than the molecular size for $\mu^{*}$ corresponding to the stability region of the periodic phase on the GS $\left(-2 / 3<\mu^{*}<14 / 3\right.$ for $\left.J^{*}=3\right)$. In Fig. 11 , we show that for $\mu^{*}=2$, where $\xi$ takes the maximum, $\Psi(L) \sim 0.1 k_{B} T$ even for system sizes 4 orders of magnitude larger than the particle diameter.
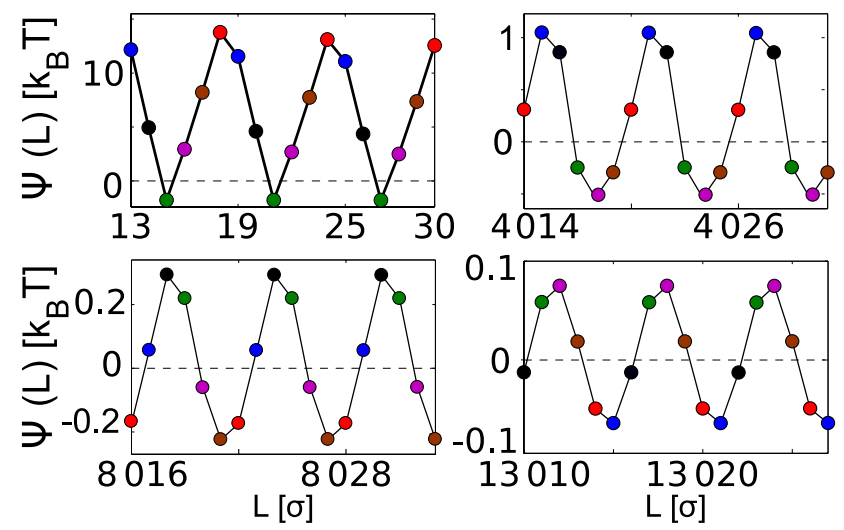

FIG. 11. $\Psi(L)$ for $J^{*}=3, T^{*}=0.2$, and $\mu^{*}=2$ for non-interacting walls. $L$ is in units of the particle diameter $\sigma$.

\section{0}

FIG. 12. Illustration of the system with elastic walls with the spring constant $k$.

\section{B. The case of elastic confining walls}

We assume that the width $L$ of the slit can oscillate around $L=L_{0}$, where $L_{0}$ is the equilibrium width in the absence of particles inside the slit. This oscillation can be controlled by a harmonic potential energy $U_{w}(L)=k \cdot\left(L-L_{0}\right)^{2}$ (see Fig. 12). Next we assume that when the slit is in contact with the reservoir of particles, and the chemical potential $\mu^{*}$ and temperature $T^{*}$ are fixed, then in mechanical equilibrium the sum of $U_{w}(L)$ and the particle-induced effective potential $\Psi(L)$ takes the minimum. We should note that similar assumptions lead to correct prediction of swelling of microporous carbons induced by adsorption of argon. ${ }^{32}$ Here we make a similar assumption for larger particles and system sizes, and softer confining surfaces. In Fig. 13, we present the sum of $\Psi$ and $U_{w}$ as a function of the system size. Note that when $L_{0}$ corresponds to the maximum of $\Psi(L)$, i.e., to a large stress induced by the confined fluid, then $U_{w}(L)+\Psi(L)$ may have two minima of very similar depth for wall separations that differ approximately by the period of the bulk structure. The number of clusters in these two states differs by one. As can be seen in Fig. 13, the barrier between the two minima is of the order of $k_{B} T$ for the assumed elastic constant $k=0.1 k_{B} T / \sigma^{2}$.

In Fig. 14, we show how the bistability appears when the chemical potential changes from $\mu^{*}=0$ or $\mu^{*}=4$ towards $\mu^{*}=2$. The barrier between the two minima decreases for increasing $\left|\mu^{*}-2\right|$. Thus, by changing the concentration of particles in the surroundings, we can change the height of the barrier and induce or suppress the jumps between the two widths of the confined system.

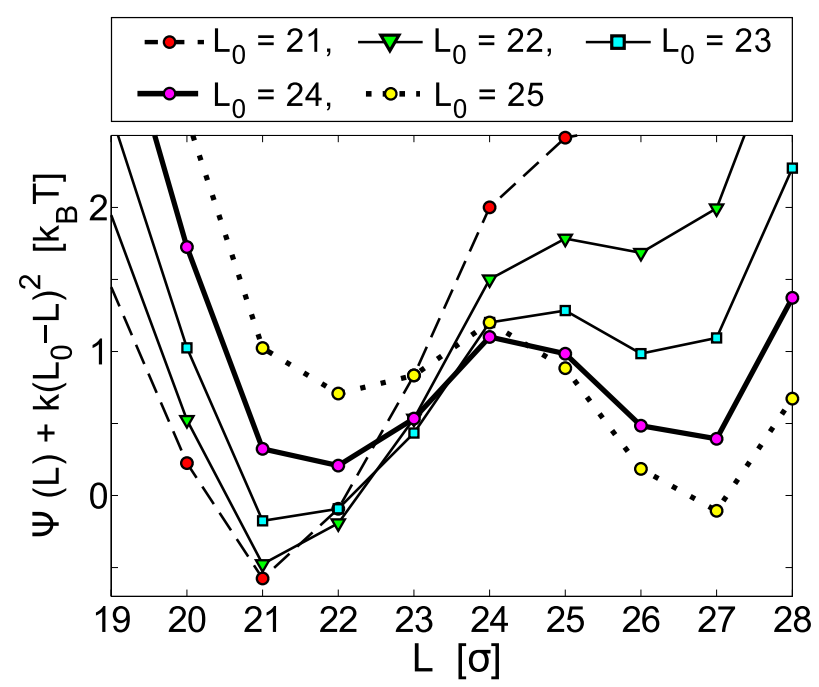

FIG. 13. The sum of the elastic energy of the confining boundaries, and the effective interaction induced by the confined self-assembling system for different equilibrium widths of the empty slit $L_{0} . J^{*}=3, T^{*}=0.5, \mu^{*}=2$, $h_{1}^{*}=h_{L}^{*}=-1$, and the spring constant $k=0.1 k_{B} T / \sigma^{2}$, where $\sigma$ is the particle diameter. 

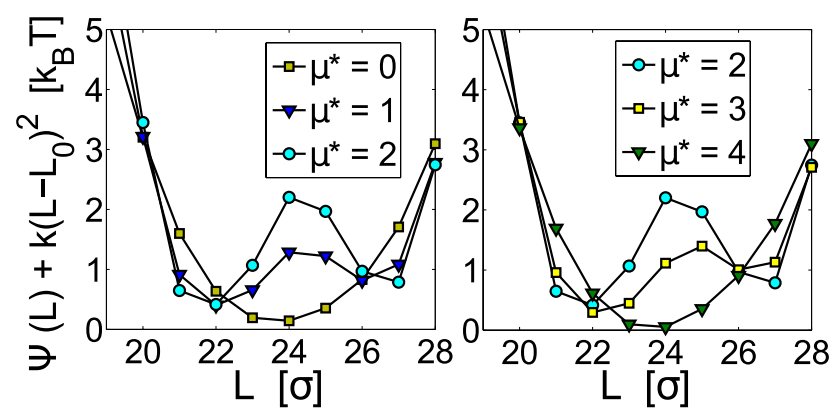

FIG. 14. The sum of the elastic energy of the confining boundaries and the effective interaction induced by the confined self-assembling system for various values of $\mu^{*} . J^{*}=3, T^{*}=0.5, h_{1}^{*}=h_{L}^{*}=-1, L_{0}=24$, and the spring constant $k=0.1 k_{B} T / \sigma^{2}$.

\section{SUMMARY}

We have solved exactly the $1 \mathrm{~d}$ model of a system interacting with the SALR potential in slits of various widths with electrically neutral surfaces. We assumed that the strengths and the ranges of the two competing parts of the potential are such that small clusters are formed. The distribution of the particles in confinement and the effective potential between the confining surfaces have been calculated for different values of the chemical potential, from dilute to dense systems in the bulk. We paid particular attention to $\mu^{*}$ corresponding to inhomogeneous distribution of the particles in the bulk. We also obtained $\rho\left(\mu^{*}\right)$ for various system sizes and different short-range interactions with the surfaces. We paid particular attention to the system sizes incommensurate with the typical distance between the clusters or layers in the bulk.

Our most interesting result is the bistability of the system confined by elastic walls (Fig. 13). The bistability occurs when the wall separation in the absence of particles corresponds to the largest stress in the confined self-assembling system. The system chooses $n$ or $n+1$ confined clusters with almost equal probability. Similar phenomenon occurs in very narrow slits when the width is such that $n$ and $n+1$ atomic layers of the adsorbed gas are equally probable. The size difference between $n$ and $n+1$ clusters is similar to the period of the bulk structure. The period is determined by the range of attractive and repulsive parts of the effective pair interaction potential and in the case of colloids can be as large as hundreds of nanometers or even micrometers. We expect that the larger is the period, the greater is the distance between the two almost equally probable system sizes. Apart from that, the bistability phenomenon should not depend on the specific shape of the effective interparticle potential as long as it leads to formation of relatively small clusters. On the other hand, we cannot exclude the possibility that for large clusters the fluctuations of the cluster size can suppress the bistability effect.

The confined self-assembling system behaves as a soft elastic material itself (Fig. 10), and the bistability takes place when its elastic constant is similar to the elastic constant of the boundaries. Such soft boundaries are formed, in particular, by biological membranes. On the other hand, if the elastic constant of the boundaries is very small, then the system can accommodate to the bulk structure and hence, the effects related with the incommensurability or particles interactions with the confinement are softened or even they vanish.

An interesting property is the possibility of inducing or suppressing the bistability by changing the chemical potential, i.e., the concentration of particles in the surroundings. The conditions in the surroundings influence also the deformations in the confined system. We found that by changing $\mu^{*}$, we induce changes in the number and size of the layers in the confined system. These structural changes are reflected in "steps" in $\rho(\mu)$. In order to help to interpret simulation results, we obtained exact expressions for $\rho\left(\mu^{*}\right)$ in the case of PBC and various system sizes. We obtained steps in $\rho\left(\mu^{*}\right)$ corresponding to the change of the type of defects resulting from the incommensurability. In order to avoid misinterpretation of these steps as phase transitions, one should verify if the steps disappear for some (commensurate) system sizes, and if their heights decays as $\sim 1 / L$ for increasing system size $L$.

We expect that different shapes of the SALR potential will lead to similar results, provided that the clusters contain only a few particles. In the case of the SALR potential leading to large clusters, as those studied in Ref. 33, the effects of confinement may be different because of the cluster-size fluctuations. This expectation is based on the observation that quite different effects of confinement were observed in the case of smalland large-periods of the bulk structure in systems containing amphiphilic molecules. ${ }^{21}$ Recently, close similarity between the bulk properties of the SALR and the amphiphilic systems has been demonstrated in Refs. 34 and 35. Based on this similarity, we may expect that the effects of confinement in the SALR and in the amphiphilic systems are similar too, but this expectation should be verified.

We should also note that our exact results concern open systems in contact with a particle reservoir. Recently, hard discs confined by a ring of particles trapped in holographic optical tweezers, which form a flexible elastic wall, were investigated. ${ }^{36,37}$ For a fixed number of confined particles, a bistable state of a hexagonal structure and concentrically layered fluid mimicking the shape of the confinement was found. This phenomenon has some similarity to our bistability, since in both cases the adaptive confinement plays a crucial role. However, the fixed number of confined particles may alter the properties of the system confined between adaptive boundaries. Some of the lipid bilayers in living cells are permeable for proteins, while some others are not. In a forthcoming paper, we shall compare the open and closed confined systems with the same average number of particles.

Finally, we would like to mention that our study of effects of boundary conditions on incommensurate structures in softmatter systems applies also to magnetic systems with nearestneighbor ferromagnetic and third neighbor antiferromagnetic interaction in a presence of magnetic field. The model is similar to the ANNNI model which is canonical in statistical physics, but previously mainly the bulk properties and the role of the coupling-constant ratio were investigated. ${ }^{38-41}$

\section{ACKNOWLEDGMENTS}

A.C. acknowledges the financial support by the National Science Center Grant No. 2012/05/B/ST3/03302. J.P. 
acknowledges the financial support by the National Science Center under Contract Decision No. DEC-2013/09/N/ST3/ 02551. N.G.A acknowledges financial support from the Dirección General de Investigación Científica y Técnica under Grant Nos. FIS2010-15502 and FIS2013-47350-C5-4-R. J.P. received funding for the preparation of the doctoral dissertation from the National Science Center in the funding of Ph.D. scholarships on the basis of the decision number DEC2014/12/T/ST3/00647.

\section{APPENDIX A: PARTITION FUNCTION}

Since the range of particle-particle interactions is 3 , we introduce boxes consisting of three neighboring lattice sites. For the system of size $L=3 N+j$, where $j=0,1,2$, the boxes can be labeled by integer $k=1,2, \ldots N$. The microstates in the $k$ th box are

$$
\hat{S}(k)=(\hat{\rho}(3 k-2), \hat{\rho}(3 k-1), \hat{\rho}(3 k)) .
$$

For $N>1(L \geq 6)$, the Hamiltonian can be written in the form

$$
\begin{aligned}
H^{*}[\{\hat{\rho}\}]= & \hat{\rho}(1) h_{1}^{*}+\hat{\rho}(L) h_{L}^{*}+H_{j}^{*}[\hat{S}(N)] \\
& +\sum_{k=1}^{N-1} H_{t}^{*}[\hat{S}(k), \hat{S}(k+1)]
\end{aligned}
$$

where

$$
\begin{aligned}
H_{t}^{*}[\hat{S}(k), \hat{S}(k+1)]= & \sum_{x=3 k-2}^{3 k}[-\hat{\rho}(x) \hat{\rho}(x+1) \\
& \left.+J^{*} \hat{\rho}(x) \hat{\rho}(x+3)-\mu^{*} \hat{\rho}(x)\right]
\end{aligned}
$$

contains the interaction between two neighboring boxes and the chemical potential term in the first box,

$$
\begin{aligned}
& H_{j}^{*}[\hat{S}(N)] \\
& = \begin{cases}-\left(\sum_{i=0}^{1} \hat{\rho}(3 N-i) \hat{\rho}(3 N-i-1)\right) & \\
-\mu^{*}\left(\sum_{i=0}^{2} \hat{\rho}(3 N-i)\right) & \text { if } j=0, \\
-\left(\sum_{i=0}^{2} \hat{\rho}(3 N+1-i) \hat{\rho}(3 N-i)\right) & \\
+J^{*} \hat{\rho}(3 N-2) \hat{\rho}(3 N+1) & \text { if } j=1, \\
-\mu^{*}\left(\sum_{i=0}^{3} \hat{\rho}(3 N+1-i)\right) & \\
-\left(\sum_{i=0}^{3} \hat{\rho}(3 N+2-i) \hat{\rho}(3 N+1-i)\right) & \text { if } j=2, \\
+J^{*}\left(\sum_{i=0}^{1} \hat{\rho}(3 N-2+i) \hat{\rho}(3 N+1+i)\right) & \\
-\mu^{*}\left(\sum_{i=0}^{4} \hat{\rho}(3 N+1-i)\right) & \end{cases}
\end{aligned}
$$

contains the particle-particle interactions between the particles which occupy the sites within the $N$ th box, and in addition the interactions between the particles at the sites labeled $3 N+1$ and $3 N+2$ (if such sites exist for given $L$ ). Finally, $\rho(1) h_{1}^{*}$ and $\rho(L) h_{L}^{*}$ are the energies of interaction between the particles and the two walls. For $N=1$, the Hamiltonian does not contain the last term in (A2). We consider only $N>1$ in this work. In order to find the partition function of the system, we introduce a $8 \times 8$ transfer matrix $\mathbf{T}$ with the matrix elements

$$
\mathbf{T}(\hat{S}(k), \hat{S}(k+1)) \equiv e^{-\beta^{*} H_{t}^{*}[\hat{S}(k), \hat{S}(k+1)]} .
$$

The partition function in terms of the transfer matrix has the following form:

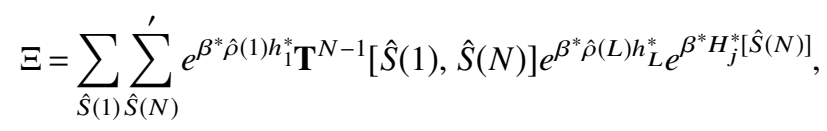

where $\sum_{\hat{S}(N)}^{\prime}$ denotes

$$
\sum_{\hat{S}(N)}^{\prime}= \begin{cases}\sum_{\hat{S}(N)} \sum_{\hat{S}(N)} \sum_{\hat{\rho}(3 N+1)} & \text { if } j=0 \\ \sum_{\hat{S}(N)} \sum_{\hat{\rho}(3 N+1)} \sum_{\hat{\rho}(3 N+2)} & \text { if } j=1 .\end{cases}
$$

We transfer $\mathbf{T}$ to the base in which it is diagonal and the matrix elements of $\mathbf{T}^{N-1}$ can be easily expressed by the sum over the eigenvalues $\lambda_{k}$ and the matrix elements $P_{k}(\hat{S}(n))$ of the matrix transforming $\mathbf{T}$ to its eigenbasis

$$
\mathbf{T}^{N-1}(\hat{S}(n), \hat{S}(m))=\sum_{k=1}^{8} P_{k}(\hat{S}(n)) \lambda_{k}^{N-1} P_{k}^{-1}(\hat{S}(m)) .
$$

Hence, the partition function is

$$
\begin{aligned}
\Xi= & \sum_{\hat{S}(1)} \sum_{\hat{S}(N)}^{\prime} \sum_{k=1}^{8} e^{\beta^{*} \hat{\rho}(1) h_{1}^{*}} P_{k}(\hat{S}(1)) \lambda_{k}^{N-1} P_{k}^{-1}(\hat{S}(N)) \\
& \times e^{\beta^{*} \hat{\rho}(L) h_{L}^{*}} e^{\beta^{*} H_{j}^{*}[\hat{S}(N)]} .
\end{aligned}
$$

\section{APPENDIX B: AVERAGE DENSITY AT A GIVEN SITE}

The framework of the transfer matrix allows us to find a formula for average density at the site $x=3 n+l$, where $n$ is the number of the triple to which the $x$ th site belongs and $l=1,2,3$ is the label of the site within the triple. For $1<n<N$, the average density at the $x$ th site is

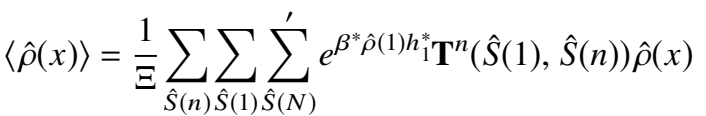

$$
\begin{aligned}
& \times \mathbf{T}^{N-(n+1)}(\hat{S}(n), \hat{S}(N)) e^{\beta^{*} \hat{\rho}(L) h_{L}^{*}} e^{\beta^{*} H_{j}^{*}[\hat{S}(N)]} .
\end{aligned}
$$

In terms of the eigenvalues it takes the form

$$
\begin{aligned}
& \langle\hat{\rho}(x)\rangle=\frac{1}{\Xi} \sum_{\hat{S}(n)} \hat{\rho}(x)\left(\sum_{\hat{S}(1)} e^{\beta^{*} \hat{\rho}(1) h_{1}^{*}} \sum_{k=1}^{8} P_{k}(\hat{S}(1)) \lambda_{k}^{n} P_{k}^{-1}(\hat{S}(n))\right) \\
& \left(\sum_{\hat{S}(N)}^{\prime} e^{\beta^{*} \hat{\rho}(L) h_{L}^{*}} e^{\beta^{*} H_{j}^{*}(\hat{S}(N))} \sum_{k=1}^{8} P_{k}(\hat{S}(n)) \lambda_{k}^{N-(n+1)} P_{k}^{-1}(\hat{S}(N))\right) .
\end{aligned}
$$

We introduce the notation

$$
\begin{aligned}
& a_{k}+i b_{k} \equiv \sum_{\hat{S}(1)} e^{\beta^{*} \hat{\rho}(1) h_{1}^{*}} P_{k}(\hat{S}(1)) P_{k}^{-1}(\hat{S}(n)), \\
& c_{k}+i d_{k} \equiv \sum_{\hat{S}(N)}^{\prime} e^{\beta^{*} \hat{\rho}(L) h_{L}^{*}} e^{\beta H_{j}^{*}(\hat{S}(N))} P_{k}(\hat{S}(n)) P_{k}^{-1}(\hat{S}(N)),
\end{aligned}
$$


where $\lambda_{1} \in \mathbf{R}$ is the eigenvalue with the largest absolute value and $i=\sqrt{-1}$. The dependence of $a_{k}, b_{k}, c_{k}$, and $d_{k}$ on $\hat{S}(n)$ is not indicated for clarity of notation. The parameters $c_{k}$ and $d_{k}$ depend also on $j=L \bmod 3$. Then, Eq. (B2) takes the form

$$
\begin{aligned}
\langle\hat{\rho}(3 n+l)\rangle= & \frac{\lambda_{1}^{N-1}}{\Xi} \sum_{\hat{S}(n)} \hat{\rho}(3 n+l)\left(\sum_{k=1}^{8}\left(\frac{\lambda_{k}}{\lambda_{1}}\right)^{n}\left(a_{k}+i b_{k}\right)\right) \\
& \times\left(\sum_{k=1}^{8}\left(\frac{\lambda_{k}}{\lambda_{1}}\right)^{N-n-1}\left(c_{k}+i d_{k}\right)\right) .
\end{aligned}
$$

Our aim is to obtain an asymptotic expression for $\langle\hat{\rho}(x)\rangle$ for $N \rightarrow \infty$ and $n \sim N / 2$. We sort the eigenvalues in the descending order of their absolute values and neglect in Eq. (B2) all the eigenvalues except from the first 3 of them. We limit ourselves to the two cases: (1) $\lambda_{2}=\bar{\lambda}_{3}=\left|\lambda_{2}\right| e^{i \lambda}$ and (2) $\lambda_{2}, \lambda_{3} \in \mathbf{R}$ with $\left|\lambda_{3} / \lambda_{2}\right|^{n} \ll 1$ for $n \gg 1$.

If $\lambda_{2}=\bar{\lambda}_{3}$, then after some algebra we obtain

$$
\begin{aligned}
\langle\hat{\rho}(3 n+l)\rangle \simeq & \frac{\lambda_{1}^{N-1}}{\Xi} \sum_{\hat{S}(n)} \hat{\rho}(3 n+l)\left(a_{1} c_{1}+2 c_{1}\left(\frac{\left|\lambda_{2}\right|}{\lambda_{1}}\right)^{n}\right. \\
& \times\left(a_{2} \cos (n \lambda)-b_{2} \sin (n \lambda)\right) \\
& +2 a_{1}\left(\frac{\left|\lambda_{2}\right|}{\lambda_{1}}\right)^{N-n-1}\left(c_{2} \cos ((N-n-1) \lambda)\right. \\
& \left.\left.-d_{2} \sin ((N-n-1) \lambda)\right)\right) .
\end{aligned}
$$

In deriving (B6) we took into account that $\left(\frac{\left|\lambda_{2}\right|}{\lambda_{1}}\right)^{n} \cdot\left(\frac{\left|\lambda_{2}\right|}{\lambda_{1}}\right)^{(N-n-1)}$ $\ll\left(\frac{\left|\lambda_{2}\right|}{\lambda_{1}}\right)^{N-n-1}$ for $N \gg 1$ and $n \sim N / 2$. Equation (B6) can be written in the form (7) with $\xi$ defined in Eq. (8), $\lambda$ defined below Eq. (8), and with the following expressions for the remaining parameters:

$$
\begin{aligned}
& \bar{\rho} \equiv \frac{\lambda_{1}^{N-1}}{\Xi} \sum_{\hat{S}(n)} \hat{\rho}(3 n+l) a_{1} c_{1}, \\
& A_{1}(l)=\left\{\begin{array}{ll}
w_{2} & \text { if } \lambda_{2}, \lambda_{3} \in \mathbf{R} \text { and }\left|\lambda_{3} / \lambda_{2}\right|^{n} \ll 1 \\
\frac{w_{2}}{\cos \theta_{1}(l)} & \text { if } \lambda_{2}=\bar{\lambda}_{3}
\end{array},\right. \\
& A_{L}(l)=\left\{\begin{array}{ll}
w_{4} & \text { if } \lambda_{2}, \lambda_{3} \in \mathbf{R} \text { and }\left|\lambda_{3} / \lambda_{2}\right|^{n} \ll 1 \\
\frac{w_{4} \exp (3 / \xi)}{\cos \theta_{L}(l)} & \text { if } \lambda_{2}=\bar{\lambda}_{3}
\end{array}\right. \text {, } \\
& \theta_{1}(l) \equiv \arctan \frac{w_{3}}{w_{2}}, \quad \theta_{L}(l) \equiv \arctan \frac{w_{5}}{w_{4}}-\lambda,
\end{aligned}
$$

where

$$
\begin{aligned}
& w_{2} \equiv \frac{2 \lambda_{1}^{N-1}}{\Xi} \sum_{\hat{S}(n)} \hat{\rho}(3 n+l) a_{2} c_{1}, \\
& w_{3} \equiv \frac{\lambda_{1}^{N-1}}{\Xi} \sum_{\hat{S}(n)} \hat{\rho}(3 n+l) b_{2} c_{1}, \\
& w_{4} \equiv \frac{2 \lambda_{1}^{N-1}}{\Xi} \sum_{\hat{S}(n)} \hat{\rho}(3 n+l) a_{1} c_{2}, \\
& w_{5} \equiv \frac{2 \lambda_{1}^{N-1}}{\Xi} \sum_{\hat{S}(n)} \hat{\rho}(3 n+l) a_{1} d_{2} .
\end{aligned}
$$

The above asymptotic expressions are not valid when $\lambda_{2}$ and $\lambda_{3}$ are both real, and $\left|\lambda_{3} / \lambda_{2}\right|^{n}=O(1)$. For the range of parameters studied in this article, however, $\lambda_{2}$ and $\lambda_{3}$ are complex conjugate numbers.

\section{APPENDIX C: SURFACE TENSION AND EFFECTIVE INTERACTION BETWEEN THE CONFINING WALLS}

The grand thermodynamic potential for the bulk system of the size $L=3 N+j$, where $j=0,1,2$, and $N \rightarrow \infty$ is

$$
\beta \Omega_{b u l k} \simeq_{N \rightarrow \infty}-\frac{L}{3 N} \ln \lambda_{1}^{N}=-\ln \lambda_{1}^{N}-\frac{j}{3} \ln \lambda_{1} .
$$

The impact of the system geometry and the particle-wall interactions can be expressed by an excess grand potential $\Omega_{e x} \equiv \Omega-\Omega_{\text {bulk }}$. We obtain the grand potential $\Omega$ of the confined system using Eq. (A7),

$$
\beta \Omega=-\ln \Xi=-\ln \left(\sum_{k=1}^{8} \lambda_{k}^{N-1} C_{k}(j)\right),
$$

where

$$
\begin{aligned}
C_{k}(j)= & \sum_{S(1)} \sum_{\hat{S}(N)}^{\prime} e^{\beta^{*} \hat{\rho}(1) h_{1}^{*} P_{k}(\hat{S}(1)) P_{k}^{-1}(\hat{S}(N))} \\
& \times e^{\beta^{*} \hat{\rho}(L) h_{L}^{*} e^{\beta^{*} H_{j}^{*}(\hat{S}(N))} .}
\end{aligned}
$$

From (C1)-(C3) we obtain

$$
\beta \Omega_{e x} \simeq \frac{3+j}{3} \ln \lambda_{1}-\ln C_{1}(j)-\ln \left(1+\sum_{k=2}^{8} \frac{C_{k}(j)}{C_{1}(j)}\left(\frac{\lambda_{k}}{\lambda_{1}}\right)^{N-1}\right) \text {. }
$$

The sum of the surface tensions and the effective potential between the confining surfaces in Eq. (6) are given by

$$
\beta\left(\gamma_{1}+\gamma_{2}\right)=\ln \lambda_{1}-\ln C_{1}(0)
$$

and

$$
\beta \Psi(L)=-\ln \left(1+\sum_{k=2}^{8} \frac{C_{k}(j)}{C_{1}(j)}\left(\frac{\lambda_{k}}{\lambda_{1}}\right)^{N-1}\right),
$$

respectively, since we have verified that the sum of the first two terms in Eq. (C4) does not depend on $j$. In the asymptotic region of $N \rightarrow \infty$, the above expression for $\Psi(L)$ takes the asymptotic form given in Eq. (9) with $\phi(j)=\phi_{2}(j)-\lambda$ and with

$$
A(j)=\left\{\begin{array}{ll}
C_{2}^{a}(j) e^{3 / \xi} & \text { if } \lambda_{2}, \lambda_{3} \in \mathbf{R} \text { and }\left|\lambda_{3} / \lambda_{2}\right|^{N} \ll 1 \\
2 C_{2}^{a}(j) e^{3 / \xi} & \text { if } \lambda_{2}=\bar{\lambda}_{3}
\end{array},\right.
$$

where $C_{k}^{a}(j) e^{i \phi_{k}(j)}=-\frac{C_{k}(j)}{C_{1}(j)}$.

\footnotetext{
${ }^{1}$ A. Stradner, H. Sedgwick, F. Cardinaux, W. Poon, S. Egelhaaf, and P. Schurtenberger, Nature 432, 492 (2004).

${ }^{2}$ A. I. Campbell, V. J. Anderson, J. S. van Duijneveldt, and P. Bartlett, Phys. Rev. Lett. 94, 208301 (2005).

${ }^{3}$ A. Shukla, E. Mylonas, E. D. Cola, S. Finet, P. Timmins, T. Narayanan, and D. I. Svergun, Proc. Natl. Acad. Sci. U. S. A. 105, 5075 (2008).

${ }^{4}$ A. J. Archer, Phys. Rev. E 78, 031402 (2008).

${ }^{5}$ A. Imperio and L. Reatto, J. Chem. Phys. 124, 164712 (2006).

${ }^{6}$ A. Ciach, Phys. Rev. E 78, 061505 (2008).

${ }^{7}$ A. Ciach and W. T. Góźdź, Condens. Matter Phys. 13, 23603 (2010).

${ }^{8}$ J. Toledano, F. Sciortino, and E. Zaccarelli, Soft Matter 5, 2390 (2009).

${ }^{9}$ P. Kowalczyk, A. Ciach, P. A. Gauden, and A. P. Terzyk, J. Colloid Interface Sci. 363, 579 (2011).
} 
${ }^{10}$ N. G. Almarza, J. Pȩkalski, and A. Ciach, J. Chem. Phys. 140, 164708 (2014).

${ }^{11}$ J. Pȩkalski, A. Ciach, and N. G. Almarza, J. Chem. Phys. 138, 144903 (2013).

${ }^{12}$ J. Pȩkalski, A. Ciach, and N. G. Almarza, J. Chem. Phys. 140, 114701 (2014).

${ }^{13}$ A. Imperio and L. Reatto, Phys. Rev. E 76, 040402 (2007).

${ }^{14}$ J. N. Israelachvili, D. J. Mitchell, and B. W. Ninham, J. Chem. Soc., Faraday Trans. 2 72, 1525 (1976).

${ }^{15}$ J. N. Israelachvili, Intermolecular and Surface Forces, 3rd ed. (Academic Press, 2011).

${ }^{16}$ P. Kékicheff and H. K. Christenson, Phys. Rev. Lett. 63, 2823 (1989).

${ }^{17}$ D. A. Antelmi, P. Kékicheff, and P. Richetti, J. Phys. II France 5, 103 (1995).

${ }^{18}$ M. W. Matsen and F. S. Bates, Macromolecules 29, 1091 (1996).

${ }^{19}$ M. W. Matsen, J. Chem. Phys. 106, 7781 (1997).

${ }^{20}$ M. Tasinkevych and A. Ciach, Phys. Rev. E 60, 7088 (1999).

${ }^{21}$ M. Tasinkevych and A. Ciach, J. Chem. Phys. 115, 8705 (2001).

${ }^{22}$ V. Babin, A. Ciach, and M. Tasinkevych, J. Chem. Phys. 114, 9585 (2001).

${ }^{23}$ V. Babin and A. Ciach, J. Chem. Phys. 119, 6217 (2003).

${ }^{24}$ W. T. Gozdz, J. Phys. Chem. B 109, 21145 (2005).

${ }^{25}$ B. M. Mladek, P. Charbonneau, and D. Frenkel, Phys. Rev. Lett. 99, 235702 (2007).
${ }^{26}$ N. B. Wilding and P. Sollich, Europhys. Lett. 101, 10004 (2013).

${ }^{27}$ W. Selke and M. E. Fisher, Phys. Rev. B 20, 257 (1979).

${ }^{28}$ R. Evans, J. Phys.: Condens. Matter 2, 8989 (1990).

${ }^{29}$ A. Ciach and W. T. Góźdź, Annu. Rep. Prog. Chem., Sect. C: Phys. Chem. 97, 269 (2001), and references therein.

${ }^{30}$ A. Ciach and V. Babin, J. Mol. Liq. 112, 37 (2004).

${ }^{31}$ F. Schmid and M. Schick, Phys. Rev. E 48, 1882 (1993).

${ }^{32}$ P. Kowalczyk, A. Ciach, and A. Neimark, Langmuir 24, 6603 (2008).

${ }^{33}$ A. J. Archer and N. B. Wilding, Phys. Rev. E 76, 031501 (2007).

${ }^{34}$ A. Ciach, J. Pȩkalski, and W. T. Góźdź, Soft Matter 9, 6301 (2013).

${ }^{35}$ J. Pȩkalski, P. Rogowski, and A. Ciach, "Self-assembly of lipids in water. Exact results from a one-dimensional lattice model," Mol. Phys. (published online).

${ }^{36}$ I. Williams, E. C. Oğuz, P. Bartlett, H. Löwen, and C. P. Royall, Nat. Commun. 4, 2555 (2013).

${ }^{37}$ I. Williams, E. C. Oğuz, P. Bartlett, H. Löwen, and C. P. Royall, J. Chem. Phys. 140, 104907 (2014)

${ }^{38}$ M. E. Fisher and W. Selke, Phys. Rev. Lett. 44, 1502 (1980).

${ }^{39}$ P. Bak, D. Mukamel, and S. Krinsky, Phys. Rev. Lett. 36, 52 (1976).

${ }^{40} \mathrm{~W}$. Selke, Phase Transitions and Critical Phenomena (Academic, New York, 1992), Vol. 15.

${ }^{41}$ W. Selke, Phys. Rep. 170, 213 (1988). 